\title{
LA SITUATION DU MARCHÉ DE LA CARPE EN EUROPE CENTRALE
}

\author{
Par le Docteur Emile SCHULMANN \\ Directeur de la Société anonyme pour l'exploitation des étangs hongrois.
}

Nous voici de nowveau au début des pêches d'étangs et les soucis que nous avons eus avec l'engraissement de la Carpe sont remplacés par ceux que donne son débit. Espérons que ceux-ci seront moindres que ceux-là, car, cette année, la vente doit corriger la fortune, les circonstances de la production ayant été très défavorables.

J'ai écrit constamment, depuis plusieurs années (I) que, selon mon opinion la récolte attendue serait inférieure à la précédente. Je suis heureux de constater que, pour la dernière fois, j'ai eu raison, la récolte de ig.34 ayant été en effet en diminution sur celle de 1933 , car autrement, personne ne maurait plus cru. Cette année, à renouveler mon pronostic habituel, je ne risque pas de me tromper. Quand on a souffert comme pisciculteur, pendant presque tout l'été, du manque d'cau ; quand an a été forcé de pîcher beaucoup d'étangs à la moitić de la campagne d'élevagge par suite de cette sécheresse anormale; quand enfin on voit déjà, à l'occasion, que l'élevage donne des résultats neftement insuffisants, on ne saurait douter que la récolte est faible.

Il est bien connu aussi que pour le maïs, la pomme de terre, la rave, etc., soit pour toutes les cultures qui exigent de la pluie pendant l'été, la récolte: de l'Europe Centrale est déficitaire. La cause en est également la sécheresse. Comme les étangs insuffisamment remplis ont leur surface róduite et contiennent peu de plancton, l'exploitant aurail wulu parer par le nourrissage artificiel, à l'insuffisance de l'alimentation nalurelle, mais malheureuscment une hausse angoissante des produits ałricoles ulilisés à cortte fiu. conséquence, elle aussi, des circonstances météorologiques. l'a retenu d’agir ainsi. Nous le voyons : un mal entraîne l'autre. Si au moins le carpiculteur avait eu l'espoir de vendre sa récolte à un prix, qui le dédommagerait de ses sacrifices ! Mais, où voir un indice encourageant? Le prix de la Carpe dans les années antérieures n incitait pas à jeter clans les étangs une grande quantité de denrées conteuses. Et la chaîne autarchiste des marchés étrangers ne desserrait pas son étreinte !

Le métier de devin est ingrat. Mais nous qui dirigeons des affaires, encore que dans un cercle étroit, avons la tâche de prévoir. Si quelqu'un prenait

(1) Voir Bulletin : - n० 39, Septembre 1931, p. 71; ‥- n० 51, Septembre 1932, p. $79 ;-n \cdot 65$, Novembre 1933 , p. $131 ;-$ no 77 . Novembre 1934 , p. 113. 
la prine de relire mon promostir dams mon artirle de lannée passée, il y

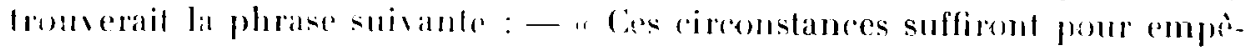
cher une momelle baisse de pris, mais des espoirs plus radien ne somient pas justifiés "( $\mathrm{I}$ ).

Et, en eflet, nous anoms un, queserpte le marché polonais, le prix de la Carpe. sur tous les marchés, a fé an moins stable. On ne duit pas oublier que cette stabilité a flé le premier hon signe après une sírie d'années qui ont apporté des chutes continurles des prix. Le devin a dome eu peurl. lan dernier, de s'avancer trop et il a eu raison, parce qu'on ne voyait pas une hausse éclatante ; à se montrer modéré dans ses espoirs, il se manifestait relativement satisfait de la situation.

Mais, en ig.3\%, nous avons eu une récolte abondante et les prix des denrées utilisées pour le notrrissage permettait un engraissement rentable. Cette année, malheureusement, on est en présence d'une faible récolte produite aver des aliments conteux. Que peut faire laugure, lui-même rarpiculteur. sinon pronostiquer des prix élevés?

Et, celte fois, ses espoirs sont motivés. T'ne hausse générale des articles de consommation se fail sentir partout. Il est vrai que la Carpe n'appartient pas ì la catégorie des comestibles de première nécessité, mais si le priv de la viande. du gihier, de la velaills augmente. les consommateurs acheteront aussi la Carpe au prix fort. Il serait hardi de compter sur un prix majoré pour la Carpe, même aver une rícolte encore pire et une production encore plus chère, si la tendance des prix de toutes les denrées alimentaires n'était franchement orienlée à la hausse. Tne aug̣mentation du prix du la seule Carpe pourrait apporter ce danger que les consommateurs renonreraient à ce poisson ; mais, dans le cas d'une hausse générale, tout te monde trowera naturel que lui aussi devienne plus cher. C'est pourquoi j'escomple, cefte année. une augmentation assez forte du prix de la Carpe.

Iprès cette introduction gémérale, passons en revue les pays de l'Europe Centrale, jouant un certain rôle. ou comme producteurs, ou comme consommateurs.

En commençant par la Hongrie, je dois dire fout d'abord que mes renscienement généraw sont en principe, tirós de non propre pays. Au moment oì j'écris ces lignes, il est encore lrop tôt pour chiffrer la réduction de la récolte de cefte année par rapport à celle de l'annéc passéc. Si le déficit sera de $20 \%$ ou plus est encore chose incertaine : ce qui est sûr. c'est qu'il Y aura un manque. Il est aussi probable que re manquue sera différent suivant les catécories de poissons. Il est surtout à rmindre (jue les sujets pesant plus de kilos soient en nombre insuffisant.

Les obstacles à lexportation ćtant devenus encore plus insurmontables qu'antérieurement, nous sommes réduits à la néeresile do développer par tous les moyens possibles la vente à l’intéricur. Fn raison do la faiblesse

(i) Bulletin, $\mathrm{n}^{\circ} 77$, Novembre $\mathbf{n} 3 \mathbf{3}$, p. irs. 
générale de la récolte. la Itongrie pourra ! plarer un plus ggrand pourcenlage de sa production.

Les efforts faits pendant des annés en ve du diveloppement de la consommation nationale ont porté leurs fruits. La Carpe, comme plat fin dans lis restaurants, a maintenu sa vogue. Quand. à Budapest. on organise un restaurant ou lorsquiun nouvel établissement ourse ses portes, sa réclame est basée sur les spécialités en poisons. la Carpe tenant le premier rangr. I raisemblablement, un srand nombre des noweaux consommatcurs a été recruté ainsi au cours des années précédentes. Quant aux prix, par suite de la faible récolte, le producteur cxige déjà une majoration. Malheureusement le Commerce ne suit pas encore. C'est au cours des prochaines semaines qu'il sera décidé si les poissonniers pourront payer plus ou si les producteurs devront se contenter des conditions de l'année passée. Personnellement, comme il a été déjà dit, je suis convaincu que les prix monteront en Hongrie.

Pour ce qui regarde la Yougcoslavic, il n'est pas encore sùr que la dimiuntion de la production y sera aussi forle quen Hongrie. Je crois, pour ma part, que la récolte ne sera pas inférieure de beauroup à la prérédente : par ailleurs, les prix des aliments artificicls n ont pas monté chez uos wisins dans la mème proportion que chez nous ; enfin l'insuffisance des pluies n'a pas été aussi accentuée. La consommation intérieure de la Yougoslavie est devenu plus forte, le marché de Belgrade surtout a fail des progrès. Cependant la Yougoslavie est forcée d'exporter encore sur unc plus vaste échelle sa récolte en Carpe. Car si la consommation du poisson r'st asscz répandue, elle absorbe une grande quantité de marée. La situation est ainsi très différente de celles de la Itongrie qui ne possède pas un port. of où la consommation des poissons est presque exclusivement réduite aux espèces d'eau douce.

La Pologne a presque perdu son importance antéricure comme débouché mour les pays exportatcurs. Il ne s'agit plus aujourd'hui des difficultés engendrées par les mesures autarchistes. Qu'on obtienne un petit contingent, fout motif manque de l'utiliser. Par exemple, au moment où j'écris, le prix moyen de la Carpe est d'environ r 40 zloty par kilogramme pour poisson rendu gare Varsovic. Or, la laxe do douane a été établie quand le prix fitait approximativement de 5 zlotys par kilogramme. Au surplus, les frais nets. sans les frais de la vente, pour expéditions de la Hongrie ou do la Yougoslavie cif. Varsovie s'élèvent à environ a zloty par kilogramme. On wit tout de suite que, dans ces conditions, l'mxportation vers la Pologne est devenue impraticable. La question est posće de savoir si l'on réussira, dans les pays exportateurs, à obtenir un redressement de cette situation. Fn tout cas, il est sûr que seule une hausse des prix en Pologne, combinée avec une réduction des droits de doune. pourra se montrer efficace.

Je ne me sens pas à même de dégager les causes de cette détérioration 
du marché polonais. Je vois seulement l'effet et ne crois pas que l'augmentation de la production polonaise puisse être le motif unique de ce changement. Il serait intéressant d'avoir sur cette question l'avis d'unc compétence.

En Allemagne, les cours sont à peu près les mèmes, que précédemment. La Carpe importée ne jouc aucun rôle dans la formation du prix, les quantités admissibles à l'importation étant très faibles au regard de la grande production allemande. La récolte de 1934 était déjà moindre que celle de l'anrée précédente ; à cause, des circonslances climatériques, la récolte de r 9.35 sern aussi - sclon l'opinion générale - médiocre. Les cours monteront sans doute ef les carpiculteurs allemands ticheront naturellement de compenser le déficit en quantité de leurs pêches par l'élévation des prix. Il faut dire que, dans les dernières annćes, lo fonctionnement du marchó i. élé très satisfaisant sans recours à des mesures brutales. Le fait que des apports trop massifs n'ont pas pesé sur lui a beaucoup facilité la marche des affaires.

Quant à I' Lutriche, j'ai déjà fait remarquer l'an dernier qu'elle fournissait un exemple de réglementation efficace. Il est vrai que la situation du marché des Carpes dans ce pays diffère de celle des autres pays de l'Europe Centrale puisque la production nationale n'est pas importante.

La situation en Tchécoslovaquie n'a gutère changé au point de vue de l'importance du poisson d'étang. Ce marché montre aussi tranquilitité et rquilibre.

Pour finir, j’insisterai une fois de plus, sur la nécessité de la propagande. Je peux constater que la conscience de cette nésessité est devenue générale partout oì la carpiculture est une branche non nógligeable de l'I griculture rationale. Sur l'initiative de l'organisation des exploitants d'étangs tchécoslovaques, on a instituí un congrès annuel des intéressés, qui a eu licu rette année à Vienne; un bon travail y a été accompli. Les délégués — parmi lespuels on royait aussi les présidents des I nions nationales de pisciculleurs et de hauts fonctionnaires d'Ftat - ont consacré plusieurs séances à cruoser les méthodes de propagande employées dians leurs pays respectifs "l ì discuter de leur efficacité. Fntre autres résolutions, il a été arrêté que les organisations nationales échangeront à l'avenir leurs informations selon des règles roncertées. Ia Tchécoslovaquie surtout a jusqu'ici obenu des résultats très satisfaisants de son activité propagandiste.

Il fut enfin décidé de tenir désormais. chaque année, une conférence de carpiculteurs dans les capitales de l'un des paýs intéressé. Pour ig.36. Budapest a élé colle désignnée.

On a recrretté à Yienne que la France ne fut pas représentée. Nous espérons tant pour nous Honcrois que pour tous les carpiculteurs de l'Europe Centrale que. l'annéc prorhaine. nous aurons le plaisir de powvoir saluer lé lélégućs français à la conférence tenue dans notre pays. 